\title{
Constraint and Mobility Change Analysis of Rubik's Cube-inspired Reconfigurable Joints and Corresponding Parallel Mechanisms
}

\author{
Duanling Li ${ }^{1,2}, \mathrm{Pu} \mathrm{Jia}^{1,3^{*}} \mathrm{D}$, Jiazhou Li $\mathrm{i}^{1,3}$, Dan Zhang ${ }^{4}$ and Xianwen Kong ${ }^{5}$
}

\begin{abstract}
The current research of reconfigurable parallel mechanism mainly focuses on the construction of reconfigurable joints. Compared with the method of changing the mobility by physical locking joints, the geometric constraint has good controllability, and the constructed parallel mechanism has more configurations and wider application range. This paper presents a reconfigurable axis ( $\mathrm{rA}$ ) joint inspired and evolved from Rubik's Cubes, which have a unique feature of geometric and physical constraint of axes of joint. The effectiveness of the $r A$ joint in the construction of the limb is analyzed, resulting in a change in mobility and topology of the parallel mechanism. The rA joint makes the angle among the three axes inside the groove changed arbitrarily. This change in mobility is completed by the case illustrated by a 3(rA)P(rA) reconfigurable parallel mechanism having variable mobility from 1 to 6 and having various special configurations including pure translations, pure rotations. The underlying principle of the metamorphosis of this $r A$ joint is shown by investigating the dependence of the corresponding screw system comprising of line vectors, leading to evolution of the $\mathrm{rA}$ joint from two types of spherical joints to three types of variable Hooke joints and one revolute joint. The reconfigurable parallel mechanism alters its topology by rotating or locking the axis of $r A$ joint to turn all limbs into different phases. The prototype of reconfigurable parallel mechanism is manufactured and all configurations are enumerated to verify the validity of the theoretical method by physical experiments.
\end{abstract}

Keywords: Reciprocal screws, Reconfigurable-axis (rA) joint, Metamorphic parallel mechanism, Constraint analysis

\section{Introduction}

In recent years, the reconfigurable mechanism [1] has become a development trend of mechanisms adapted to different requirements and environments. This has led to other developments in the kinematic mechanism and metamorphic mechanisms with the ability to change mobility.

Metamorphic mechanism [2] is derived from the concept of metamorphosis in the sense of evolutionary design. Its metamorphic principle mainly depends on the change of geometric or physical constraints, which can change its own topological structure and subsequent

\footnotetext{
*Correspondence: jiapu1990@163.com

${ }^{1}$ School of Automation, Beijing University of Posts

and Telecommunications, Beijing 100876, China

Full list of author information is available at the end of the article
}

mobility. Zhang et al. [3] analyzed the rule extension of embedded spatial model with metamorphic characteristics to develop metamorphic operations. Four types of branching singularities are identified by Gogu [4] from the new formula of mobility, connectivity, overconstraint and redundancy of parallel robots. Kong et al. [5-7] presented a method for the type synthesis of parallel mechanisms with multiple operation modes. Gan et al. [8-12] proposed three kinds of metamorphic parallel mechanisms composed of three limbs with reconfigurable Hooke joints, and the unified kinematics analysis of a new 3rTPS metamorphic parallel mechanism were investigated. Zhang et al. [13-15] proposed a kind of metamorphic parallel mechanism extracted from origami fold and analyzed the evolutionary kinematic configuration changes of two metamorphic parallel mechanisms 
of 3SvPSv. A new type of reconfigurable modular parallel robot was proposed by Carbonari et al. [16], which is equipped with a locking system to fix one of the revolute joints and is able to perform different types of motion, especially pure translation and pure rotation. Ye et al. [17-19] proposed a family of reconfigurable parallel mechanism with reconfigurable hybrid limbs based on diamond kinematic chain. The reconfigurable parallel mechanism has ability to perform variable motion modes, such as 3T, 2T1R, 2R1T and 3R. Wang et al. [20] proposed a new reconfigurable parallel mechanism based on a reconfigurable spatial five bar mechanism with two different working modes, which can realize four kinds of motions. Qu et al. [21] analyzed the topology and mobility of a novel redundant reconfigurable parallel mechanism. Wang et al. [22] investigated the application of parallel leg mechanism in Quadruped/bipedal reconfigurable walking robot. Tian et al. [23, 24] investigated the structural synthesis of a class of reconfigurable parallel mechanisms based on closed-loop metamorphic linkage and five bar metamorphic linkages. Song et al. [25] proposed a new type of $6 R$ metamorphic mechanism with eight motion branches and multiple bifurcation points, and explained the transformation of kinematic branches by kinematic curves.

The metamorphic rT joint mentioned in Ref. [8] consists of two revolute joints which are perpendicular to each other. Besides, one of the revolute joint axes can not only rotate independently as a revolute joint, but also be used to adjust the spatial position of another. However, the mobility of the joint is constant. It changes the mobility of the limb only by linear dependent with other revolute joints. Although the vA joint mentioned in Ref. [13] has ability to realize the mobility change from spherical joint, Hooke joint and revolute joint, there is only one type of equivalent motion joint, especially in the configuration of equivalent spherical joint. Since the phase of equivalent spherical joint can not form three-axis vertical spherical joint, the working space of metamorphic parallel mechanism composed of vA joint will be limited.

The $\mathrm{rA}$ joints proposed in this paper can not only alter the mobility of joint, but also the mobility of the constructed limbs and mechanisms. In addition, it formed a variety of different types of spherical joint, Hook joint and a revolute joint.

\section{Design of rA Joint}

As aforementioned, Rubik's Cube is seen in daily life in different forms including third-order cube and fourth-order cube. However, its internal structure is rarely noticed. From the reconfigurable joint point of view, three revolute-joint axes within the Rubik's Cube
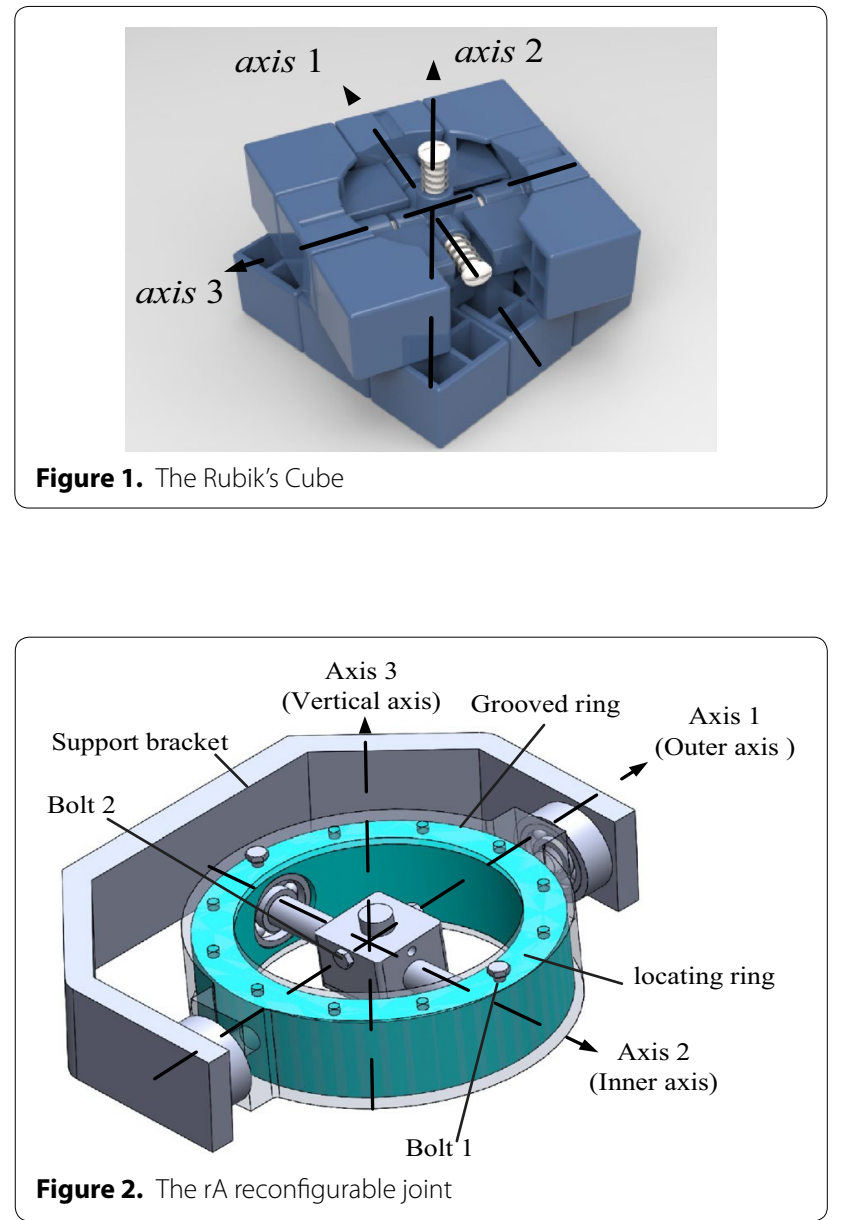

are perpendicular to each other as a standard spherical joint, and the angle between them is a constant, as shown in Figure 1.

In order to realize the conversion among spherical joint, Hooke joint and revolute joint, the relative position or motion between the three axes is changed by geometric or physical locking to form a joint with variable mobility. A reconfigurable joint is designed as shown in Figure 2.

Since the position of axis 2 of the joint is adjustable and axis 3 can be locked, the joint is named rA joint (reconfigurable axis joint). As shown in Figure 2, the rA joint consists of support bracket, grooved ring, locating ring and three revolute joints. Besides, the position of the inner axis can be adjusted around the vertical axis which can not only act as an independent revolute joint, but also adjust the position of the inner axis. When the inner axis is adjusted to a specific position, it is fixed on the grooved ring by bolts. Therefore, the inner axis can be adjusted to any position in the grooved ring. 


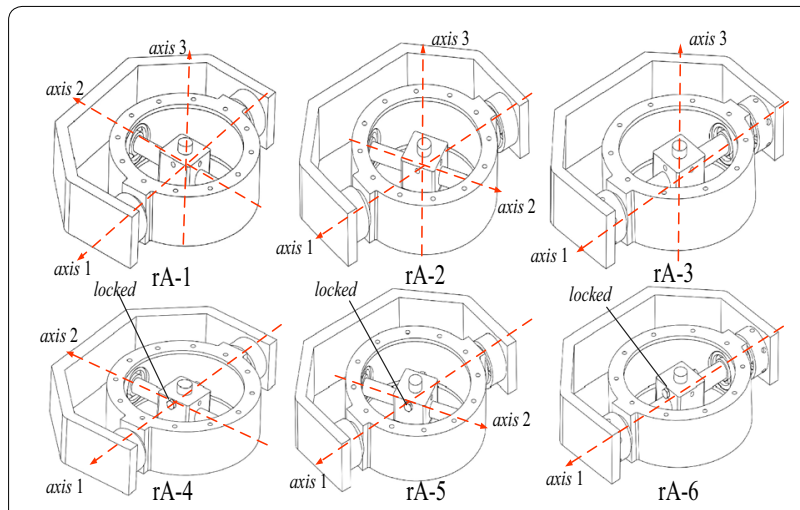

Figure 3. The six phases of $\mathrm{rA}$ joint

As shown in Figure 3, the phases of rA joint can be divided into two kinds of spherical joints, three kinds of Hooke joints and one kind of revolute joint. The phases of $\mathrm{rA}$ joints are defined as follows.

Equivalent spherical joint phases are as follows.

When axis 2 is adjusted to be perpendicular to axis 1 , the three axes of $\mathrm{rA}$ joint are perpendicular to each other. At this time, the rA joint is equivalent to a standard spherical joint and expressed as rA-1. The axis 2 is adjusted to the position where it is neither perpendicular nor collinear with the axis 1 , resulting in the rA joint in this phase being expressed as rA-2, which is an equivalent spherical joint whose three axes are not perpendicular to each other.

Equivalent Hooke joint phase: when the axis 2 is adjusted to be collinear with the axis 1 , the mobility of $\mathrm{rA}$ joint decreases from 3 to 2 , and a joint with two effective axes is generated. At this time, the rA joint is equivalent to a Hooke joint and denoted as rA-3. When the axis 3 of the $\mathrm{rA}-1$ phase is locked physically, the axis 2 and the axis 1 of the $\mathrm{rA}$ joint are the effective axes. At this time, the phase with two mutually vertical axes is named rA-4. In the same way, when the axis 3 of the rA-2 is locked, only the axis 2 and the axis 1 which are not perpendicular to each other are effective, and the phase is named as rA-5.

Equivalent revolute joint phase: when the axis 3 in $\mathrm{rA}-3$ is locked and the axis 2 and the axis 1 are collinear, the rA joint has only one DOF (degree of freedom), which is equivalent to a revolute joint composed of the axis 2 and the axis 1. The rA joint in this phase is denoted as rA-6.

According to the above definition, $r A$ joint can be divided into six phases, including two kinds of spherical joint, three kinds of Hooke joint and one revolute joint. It should be mentioned that the change of the position of the axis 2 is controlled by the axis 3 . When adjusting the position of the axis 2 , it is necessary to ensure that the

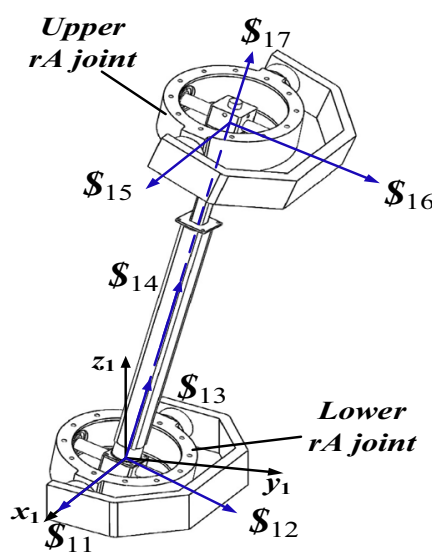

Figure 4. $(r A-1) P(r A-1) \operatorname{limb}$

axis 3 is not locked and perpendicular to the plane of the grooved ring.

\section{Phase Analysis of Reconfigurable Limb}

\subsection{Structure Design of the Reconfigurable Limb}

Taking rA joint as the module of reconfigurable unit and integrating it with a prismatic joint into the design of the limb, the change of the phases of $\mathrm{rA}$ joint leads to a series of changes of the motion mode of the limb as in Figure 4. For further analysis, the $\mathrm{rA}$ joint at the upper end and the rA joint at the lower end of the limb are represented as upper $\mathrm{rA}$ joint and lower $\mathrm{rA}$ joint respectively. In Section 3.2, limbs with specific DOF are selected as representatives, and the constraints of each phase will be analyzed.

\subsection{Constraint Analysis of the Reconfigurable Limb \\ 3.2.1 Analysis of the Unconstrained Limb Phase}

When the rA joints connected to both ends of the prismatic joint are in rA-1 as shown in Figure 4.

The motion-screw system can be given by

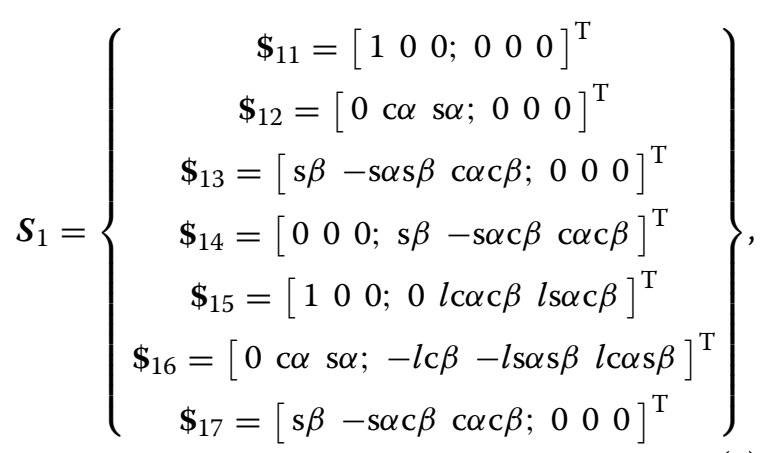

where $\alpha$ and $\beta$ are the angles between $S_{13}$ and $y_{1}$ axis, $S_{13}$ and $S_{14}$ respectively, and $l$ is the length of the limb 
measured from the origin of two rA joints. In order to simplify the equation, sine and cosine are expressed as $\mathrm{s}$ and $\mathrm{c}$ respectively. There are 7 twists in the motion system of (rA-1)P(rA-1). The limb is equivalent to SPS, where $\mathrm{S}$ represents the spherical joint and P represents the prismatic joint. The twist $S_{13}$ and $S_{17}$ are the same, resulting in that the twists system is a 6 -system. Therefore, there is no constraint delivered on the platform by the limb (rA-1) $\mathrm{P}(\mathrm{rA}-1)$.

Similarly, when either of the upper and lower RA joints is an equivalent Hooke joint, the limb is equivalent to the UPS, where $U$ represents the universal joint. Therefore, (rA-3) P (rA-1), (rA-4) P (rA-1) and (rA-5) P (rA-1) do not impose any constraint on the platform.

\subsubsection{Constraints and Phase Change of the UPU limb}

In Figure 5, the rA joints connected to both ends of the prismatic joint are in $\mathrm{rA}-4$. The limb phase is equivalent to UPU, and the motion-screw system can be given by

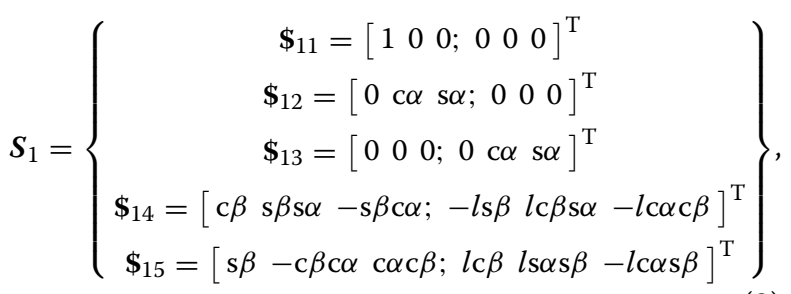

where $\alpha$ is the angle between $S_{12}$ and $y_{1}$ axis, $\beta$ is the angle between $S_{13}$ and its projection on the plane $o_{1} y_{1} z_{1}$.

The limb constraint system is given as

$$
\boldsymbol{S}^{\mathbf{r}}=\left[\begin{array}{cccccc}
0 & 0 & 0 ; & 0 & \mathrm{~s} \alpha & \mathrm{c} \alpha
\end{array}\right]^{\mathrm{T}},
$$

This constraint system shows that the limb (rA-4) P (rA-4) exerts a constraint force on the platform, and its direction is perpendicular to the prismatic joint in the local frame.

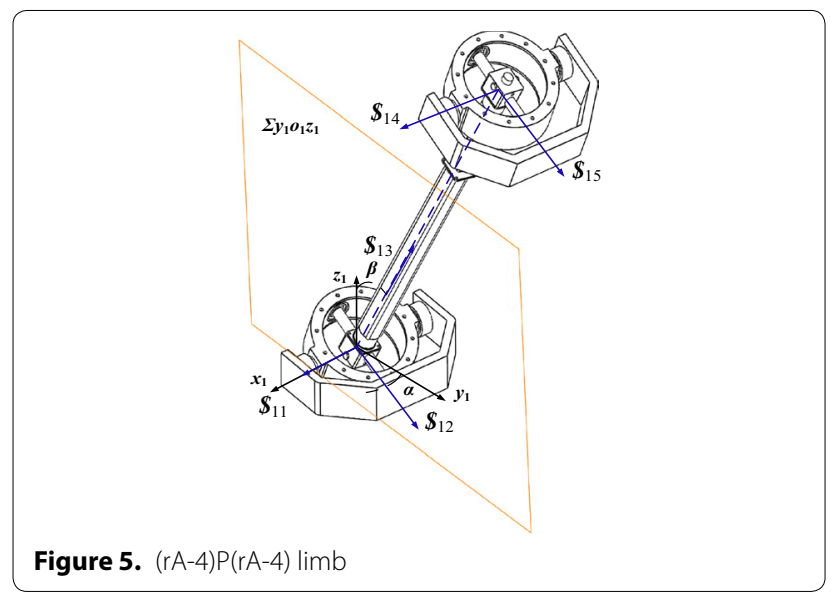

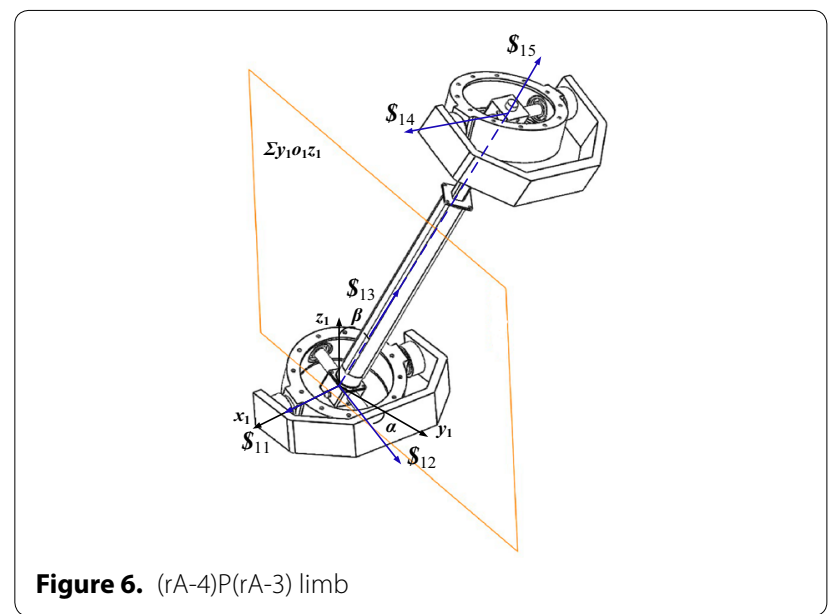

When the upper and lower $\mathrm{rA}$ joints are converted to rA-3 and rA-4 respectively, as shown in Figure 6, the limb motion-screw system is given as

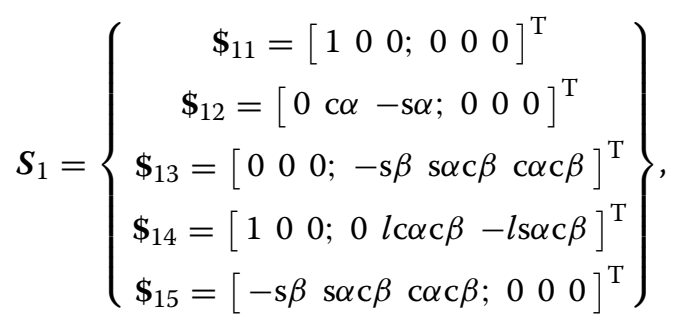

where $\alpha$ is the angle between $S_{12}$ and $y_{1}$ axis, $\beta$ is the angle between $S_{13}$ and its projection on the plane $o_{1} y_{1} z_{1}$.

The limb constraint system is given as

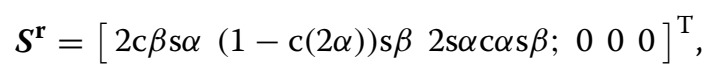

This constraint system shows that the limb (rA-4) $\mathrm{P}(\mathrm{rA}-3)$ exerts a constraint force on the platform, and its direction is perpendicular to the prismatic joint in the local frame.

When the upper and lower $\mathrm{rA}$ joints are converted to the phase of rA-3, as shown in Figure 7, limb motionscrew system is given as follows:

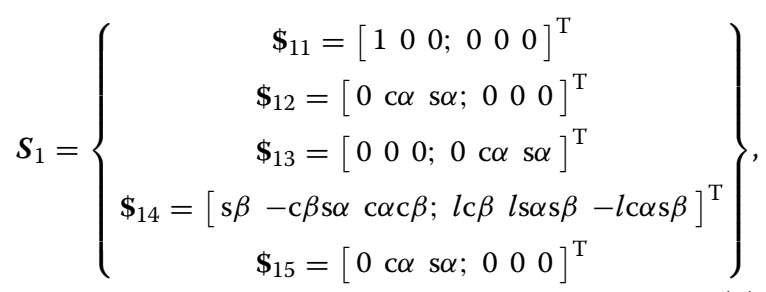

The constraint screw of limb can be obtained by calculating the reciprocal screw of the motion screw in Eq. (6), that is 


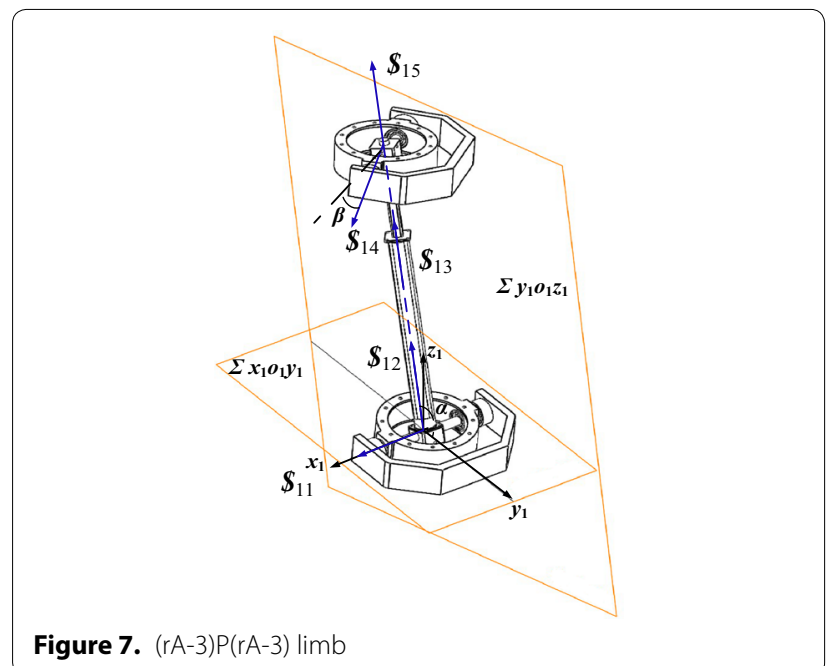

$$
\boldsymbol{S}^{\boldsymbol{r}}=\left\{\begin{array}{c}
\boldsymbol{\$}_{1}^{\mathrm{r}}=\left[\begin{array}{llllll}
1 & 0 & 0 ; & 0 & l \mathrm{~s} \alpha & -l \mathrm{c} \alpha
\end{array}\right]^{\mathrm{T}} \\
\mathbf{\$}_{2}^{\mathrm{r}}=\left[\begin{array}{lllll}
\mathrm{s} \beta & -\mathrm{s} \alpha \mathrm{c} \beta & \mathrm{c} \alpha \mathrm{c} \beta & 0 & 0
\end{array}\right]^{\mathrm{T}}
\end{array}\right\},
$$

The constraint screw system in Eq. (7) indicates that there are two constraint forces delivered on the platform. One of them passes through the center of the upper $\mathrm{rA}$ joint, whose direction is consistent with the outer axis of the lower rA joint. Another constraint force passes through the center of the lower rA joint, and the direction is consistent with the twist $S_{14}$.

\subsubsection{Constraints and Phase Change of the RPS Limb}

When the lower $\mathrm{rA}$ joint in the limb is converted into the rA-6 joint, and the upper $\mathrm{rA}$ joint is converted into the rA-1 joint, as shown in Figure 8, the limb phase is equivalent to RPS where all the commonly used revolute joints in this phase are effective.

The motion-screw system can be given as

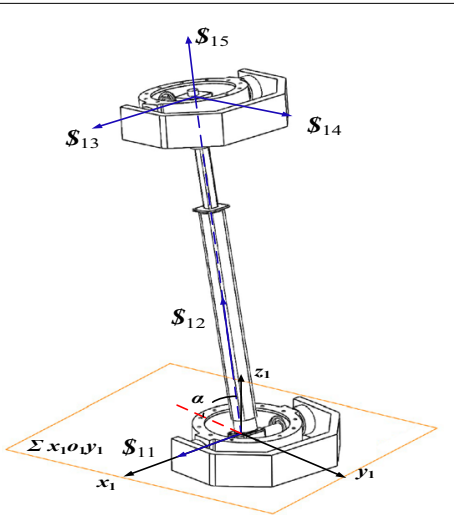

Figure 8. $(r A-6) P(r A-1) \operatorname{limb}$

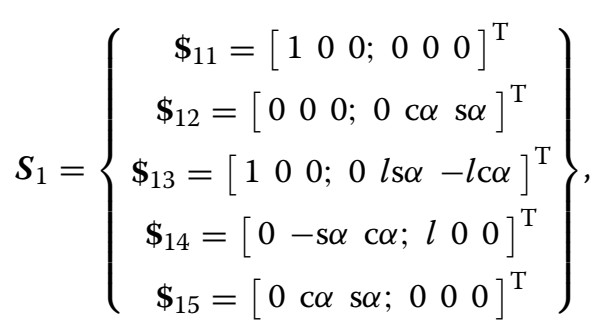

It is clear that the five twists are independent, leading to the generation of a five-system. Since the constraints provided by the reciprocal screws determine the motion of moving platform, the limb constraint system is given as

$$
\boldsymbol{S}^{\mathbf{r}}=\left[\begin{array}{llllll}
1 & 0 & 0 ; & 0 & l \mathbf{s} \alpha & -l \mathrm{c} \alpha
\end{array}\right]^{\mathrm{T}},
$$

According to Eq. (9), the limb (rA-6) $\mathrm{P}(\mathrm{rA}-1)$ has a constraint force on platform whose direction is consistent with the axis of $S_{11}$ and passes through the center of the upper rA joint.

\subsubsection{Constraints and Phase Change of the RPR Limb}

As shown in Figure 9, limb (rA-6) P(rA-1) can be changed into the phase $(\mathrm{rA}-6) \mathrm{P}(\mathrm{rA}-6)$ which is equivalent to phase RPR.

The motion-screw system is given as

$$
\boldsymbol{S}_{1}=\left\{\begin{array}{c}
\boldsymbol{\$}_{11}=\left[\begin{array}{llllll}
1 & 0 & 0 ; & 0 & 0 & 0
\end{array}\right]^{\mathrm{T}} \\
\boldsymbol{\$}_{12}=\left[\begin{array}{llllll}
0 & 0 & 0 ; & 0 & \mathrm{c} \alpha & \mathrm{s} \alpha
\end{array}\right]^{\mathrm{T}} \\
\mathbf{\$}_{13}=\left[\begin{array}{llllll}
1 & 0 & 0 & 0 & l \mathrm{~s} \alpha & -l \mathrm{c} \alpha
\end{array}\right]^{\mathrm{T}}
\end{array}\right\},
$$

The motion screw system in Eq. (10) forms a 3-system, which results in three constraints on the platform. The constraint screws system can be produced as

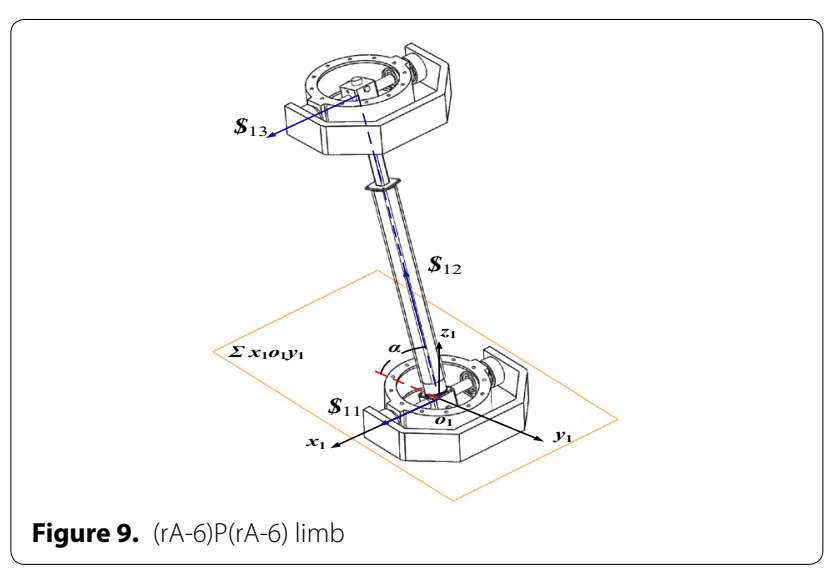




$$
\boldsymbol{S}^{\mathbf{r}}=\left\{\begin{array}{l}
\boldsymbol{\$}_{1}^{\mathrm{r}}=\left[\begin{array}{llllll}
1 & 0 & 0 & 0 & 0 & 0
\end{array}\right]^{\mathrm{T}} \\
\boldsymbol{\$}_{2}^{\mathrm{r}}=\left[\begin{array}{llllll}
0 & 0 & 0 & 0 & 1 & 0
\end{array}\right]^{\mathrm{T}} \\
\boldsymbol{\$}_{3}^{\mathrm{r}}=\left[\begin{array}{llllll}
0 & 0 & 0 & 0 & 0 & 1
\end{array}\right]^{\mathrm{T}}
\end{array}\right\},
$$

The constraint screw system in Eq. (11) provides three constraints, including one constraint and two constraint couple. The constraint force is collinear with the $x_{1}$-axis, and the two constraint couples are parallel with the $y_{1}$ and $z_{1}$-axes respectively.

\section{Constraint Analysis of 3(rA)P(rA)}

As aforementioned in Section 3, due to the diversity of the limb, the parallel mechanism has a variety of configurations, and mobility can be switched from 6 to 1 . Some special configurations such as three rotation and three translation configurations can be constructed.

\subsection{Metamorphic Parallel Mechanism 3(rA)P(rA)}

In this section, the topology and constraint analysis of the metamorphic parallel mechanism composed of three (rA)P(rA) limbs, platform and the base, are investigated. By investigating the constraints of three limbs delivered on platform, the mobility of the parallel mechanism can be obtained. Finally, the metamorphic parallel mechanism is able to form a variety of mobility configurations, which covers degrees of freedom from 6 to 1 , and including two special configurations such as 3-DOF rotational motion and 3-DOF translational motion.

\subsection{Mobility Analysis of $3(\mathrm{rA}) \mathrm{P}(\mathrm{rA})$}

Since the limb (rA)P(rA) has four different constraint phases, the parallel mechanism $3(\mathrm{rA}) \mathrm{P}(\mathrm{rA})$ with specific mobility can be constructed by the combination of limbs with different phases. Based on the constraints provided by different phases of the (rA) $\mathrm{P}(\mathrm{rA})$ limb, in order to construct a parallel mechanism with the required motion, certain geometric conditions should be met by the limbs.

All configurations of the metamorphic parallel mechanism can be divided into two types. The first is the configurations with different topologies but the same mobility, and the second is the configurations with different topologies and mobility.

\subsubsection{Reconfiguration to Mobility 6}

The local coordinate systems $\left\{x_{i} y_{i} z_{i}\right\}$ are established by rotating the global coordinate system $\phi_{i}=2(i-1) \pi / 3$ around the $Z$ axis $(i=1,2$, and 3$)$, as well as shifting it along the axis parallel to the $Y$ axis to the distance $r_{\mathrm{b}}$ from the origin of each local coordinate system, where $r_{\mathrm{b}}$ is the radius of the circumscribed circle of base, and its origin is connected to the origin to the common point $B_{i}(i=1$, 2 , and 3 ).
The constraints provided by each limb represented by a local frame $\left\{x_{i} y_{i} z_{i}\right\}$ can be obtained from the analysis in Section 3. And then convert the limb constraint screws to the global frame $O-X Y Z$, which is fixed at the center of the base by the following transformation.

$$
\boldsymbol{S}_{i}^{\mathbf{r}}=\left({ }_{i}^{0} \boldsymbol{T}\right)\left(\boldsymbol{S}_{i 1}^{\mathrm{r}}\right)^{\mathrm{T}}=\left[\begin{array}{cc}
\boldsymbol{R}\left(\phi_{i}\right) & \mathbf{0} \\
\boldsymbol{A}_{i} \boldsymbol{R}\left(\phi_{i}\right) & \boldsymbol{R}\left(\phi_{i}\right)
\end{array}\right]\left(\boldsymbol{S}_{i 1}^{\mathrm{r}}\right)^{\mathrm{T}},
$$

where

$$
\boldsymbol{A}_{i}=\left[\begin{array}{ccc}
0 & -O_{i z} & O_{i y} \\
O_{i z} & 0 & -O_{i x} \\
-O_{i y} & O_{i x} & 0
\end{array}\right] \quad \boldsymbol{R}\left(\phi_{i}\right)=\left[\begin{array}{ccc}
\mathrm{c} \phi_{i} & -\mathrm{s} \phi_{i} & 0 \\
\mathrm{~s} \phi_{i} & \mathrm{c} \phi_{i} & 0 \\
0 & 0 & 1
\end{array}\right]
$$

where $\left[O_{i x}, O_{i y}, O_{i z}\right]^{\mathrm{T}}$ is the position vector of the origin $o_{i}$ of the local coordinate system in the global coordinate system $\{X Y Z\}$.

From the previous analysis, it can be seen that when the limb is in (rA-1)P(rA-1) phase as Figure 10, the motion system of the limb is a 6-system, thus the three limbs have no constraints on the platform.

\subsubsection{Reconfiguration to Mobility 5}

By changing the phase of a limb to obtain a constraint from the unconstrained phase, a parallel mechanism with $5 \mathrm{DOF}$ can be obtained as Figure 11 .

In the parallel mechanism, limb 2 and limb 3 keep the (rA-1)P(rA-1) phase unchanged, and the lower rA joint of limb 1 turn into rA-6 phase, as shown in Figure 11. At this time, the metamorphic parallel mechanism changes to 2(rA-1) $\mathrm{P}(\mathrm{rA}-1)-1(\mathrm{rA}-6) \mathrm{P}(\mathrm{rA}-1)$ from 3(rA-1)P(rA-1). So the constraint screw system is produced as

$$
\boldsymbol{S}^{\mathbf{r}}=\left\{\begin{array}{l}
\boldsymbol{\$}_{1}^{\mathrm{r}}=\left[\begin{array}{llllll}
1 & 0 & 0 ; & 0 & l \mathrm{~s} \alpha & r_{b}-l \mathrm{c} \alpha
\end{array}\right]^{\mathrm{T}} \\
\boldsymbol{\$}_{2}^{\mathrm{r}}=\left[\begin{array}{llllll}
0 & 0 & 0 ; & 0 & 0 & 0
\end{array}\right]^{\mathrm{T}} \\
\boldsymbol{\$}_{3}^{\mathrm{r}}=\left[\begin{array}{llllll}
0 & 0 & 0 & 0 & 0 & 0
\end{array}\right]^{\mathrm{T}}
\end{array}\right\},
$$

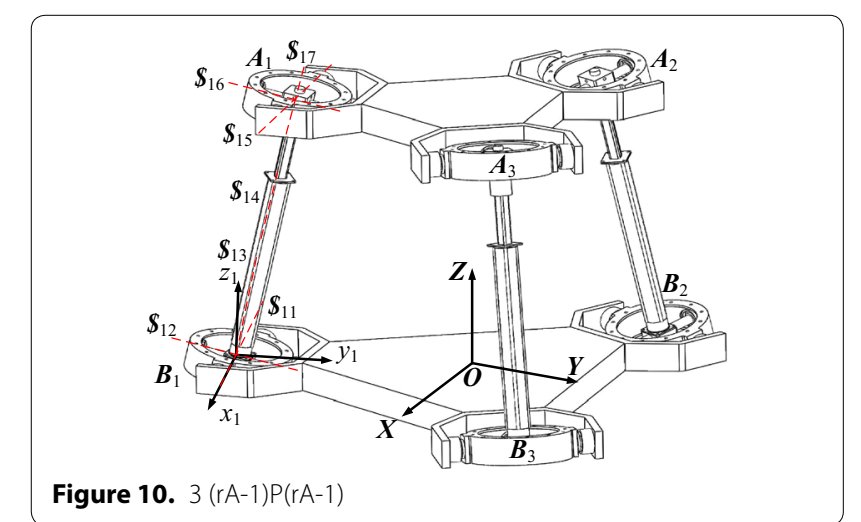




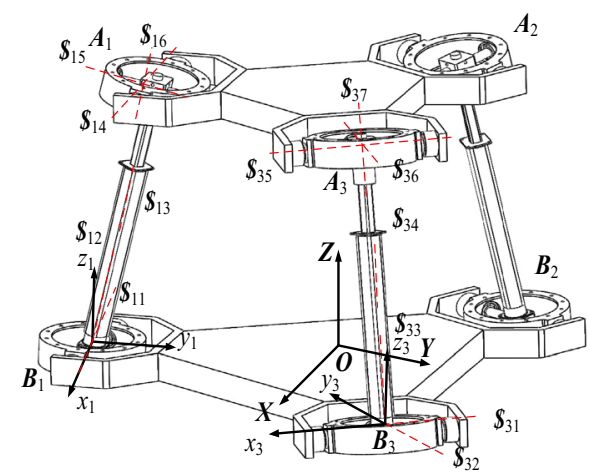

Figure 11. $1(r A-6) P(r A-1)-2(r A-1) P(r A-1)$

Since the motion screw and constrained screw are reciprocal, the motion screw can be obtained by calculating the reciprocal screw of Eq. (13):

$$
\boldsymbol{S}_{\mathbf{f}}=\left\{\begin{array}{c}
\mathbf{\$}_{f 1}=\left[\begin{array}{ccccccc}
0 & 0 & 1 ; & l \mathbf{c} \alpha & -r_{b} & 0 & 0
\end{array}\right]^{\mathrm{T}} \\
\mathbf{\$}_{f 2}=\left[\begin{array}{llllll}
0 & 1 & 0 ; & -l \mathbf{s} \alpha & 0 & 0
\end{array}\right]^{\mathrm{T}} \\
\mathbf{\$}_{f 3}=\left[\begin{array}{llllll}
1 & 0 & 0 & 0 & 0 & 0
\end{array}\right]^{\mathrm{T}} \\
\mathbf{\$}_{f 4}=\left[\begin{array}{llllll}
0 & 0 & 0 ; & 0 & 0 & 1
\end{array}\right]^{\mathrm{T}} \\
\mathbf{\$}_{f 5}=\left[\begin{array}{llllll}
0 & 0 & 0 & 0 & 1 & 0
\end{array}\right]^{\mathrm{T}}
\end{array}\right\}
$$

Based on this, the platform has five DOF, which are three rotations about $X, Y$, and $Z$ axes, and two translations along the $Y$ and $Z$ axes.

\subsubsection{Reconfiguration to Mobility 4}

Limbs 1 and 3 maintain the phase of (rA-6)P(rA-1), and have both motion twists and constraints, as shown in Eq. (8) and Eq. (9). The lower rA joint of limb 2 turn into $\mathrm{rA}-1$ phase, resulting in the limb being in the $(\mathrm{rA}-1) \mathrm{P}(\mathrm{rA}-$ 1) phase as in Figure 12.

The constraint screws are produced as

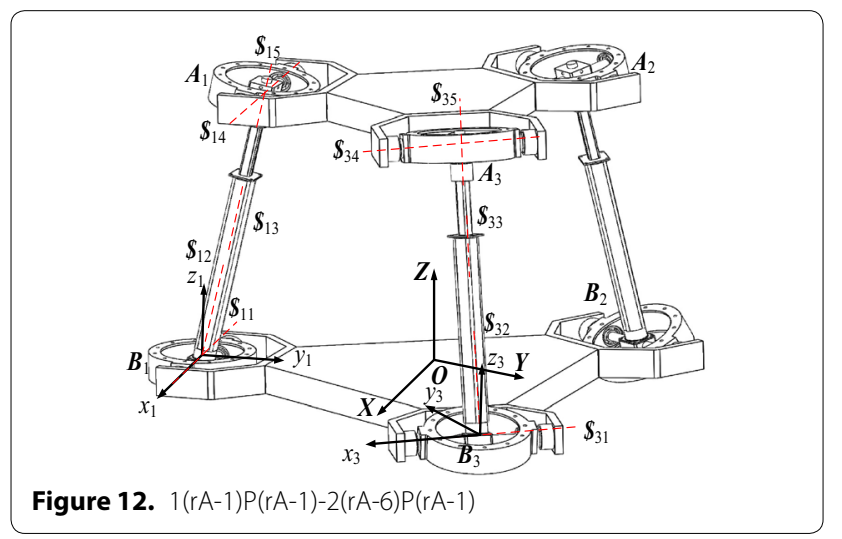

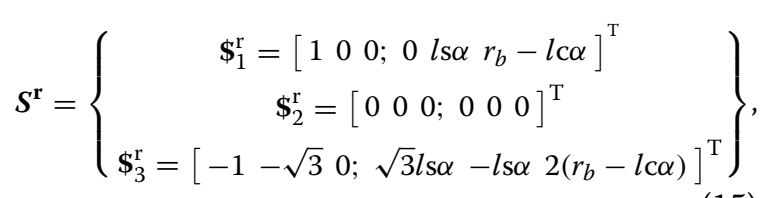

The motion screws are reciprocal to $S^{\mathrm{r}}$ and the screw system can be calculated form Eq. (15), that is

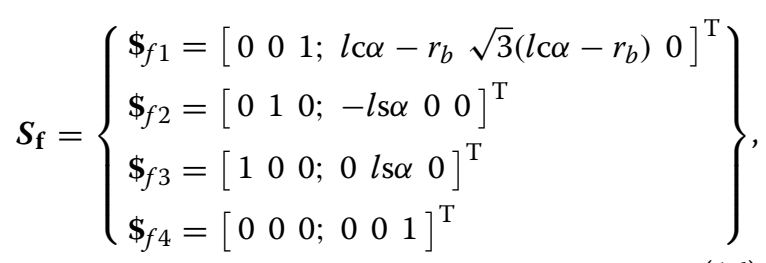

From the above equation, it can be seen that the platform has four DOF, including three rotations about the $X, Y$ and $Z$ axes and one translation along the $Z$ axis.

\subsubsection{Mobility Configuration of 3-DOF Translation}

Due to the variety of motion modes of 3UPU parallel mechanism, it has been widely studied by many scholars. 3(rA) $\mathrm{P}(\mathrm{rA})$ parallel metamorphic mechanism can be transformed into 3UPU parallel mechanism by adjusting the $\mathrm{rA}$ joint in the three limbs to the equivalent Hooke joint phase.

In general, the constraints and mobility of all parallel mechanisms with the same limb structure can be analyzed by one limb and then extended it to other limbs. Therefore, when all three limbs are in phases of (rA-4) $\mathrm{P}(\mathrm{rA}-4)$, a parallel mechanism with pure translation is assembled as Figure 13.

The analysis of $3(\mathrm{rA}-4) \mathrm{P}(\mathrm{rA}-4)$ configuration shows that there are no common constraints and redundant constraints. Each limb has no local degree of freedom, and the mobility of the mechanism is 3 . Therefore, the constraint system of the mechanism is as follows

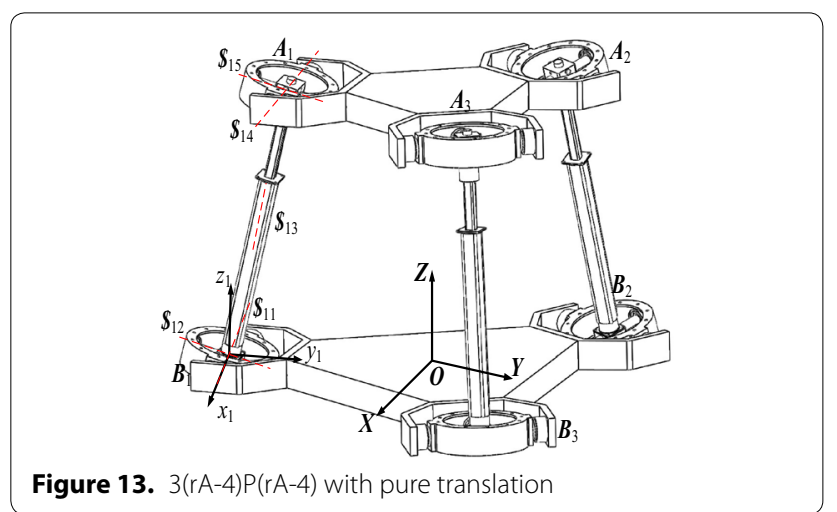




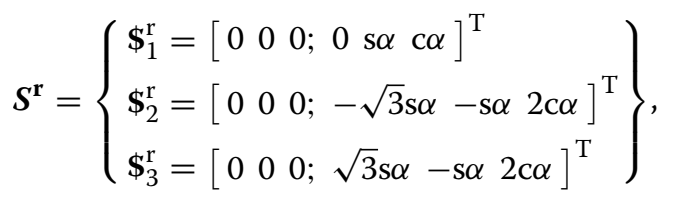

The motion screw of the mechanism is the reciprocal of $\boldsymbol{S}^{\mathrm{r}}$, and screw system can be calculated from Eq. (17):

$$
\boldsymbol{S}_{\mathbf{f}}=\left\{\begin{array}{l}
\mathbf{\$}_{f 1}=\left[\begin{array}{llllll}
0 & 0 & 0 & 1 & 0 & 0
\end{array}\right]^{\mathrm{T}} \\
\mathbf{\$}_{f 2}=\left[\begin{array}{llllll}
0 & 0 & 0 & 0 & 1 & 0
\end{array}\right]^{\mathrm{T}} \\
\mathbf{\$}_{f 3}=\left[\begin{array}{llllll}
0 & 0 & 0 & 0 & 0 & 1
\end{array}\right]^{\mathrm{T}}
\end{array}\right\}
$$

This indicates that the three rotations of the moving platform are constrained. The platform has three translational DOF along the $X, Y$, and $Z$ axes and the 3(rA-4) $\mathrm{P}(\mathrm{rA}-4)$ parallel mechanism implements pure translational motion.

\subsubsection{Mobility Configuration of 3-DOF Rotation}

According to the analysis in Figure 6, the (rA-4)P(rA-3) limb exerts a constraint force on the platform. Therefore, the 3(rA-4) $\mathrm{P}(\mathrm{rA}-3)$ parallel mechanism, which is composed of three identical (rA-4)P(rA-4) limbs, a platform and a base, has three rotational DOF.

The 3(rA-4)P(rA-3) metamorphic parallel mechanism can be evolved into a pure rotational configuration by altering the upper and lower $\mathrm{rA}$ joints of all the three limbs as in Figure 14.

Therefore, the constraint force of the remaining two limbs is:

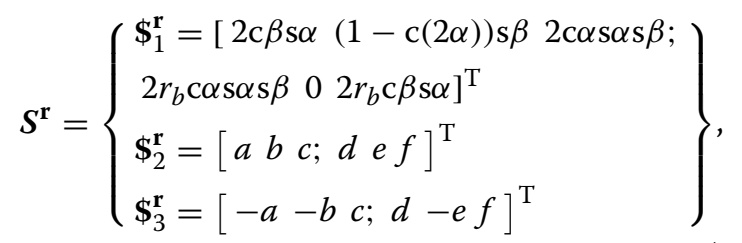

where

$$
\begin{gathered}
a=-\frac{\mathrm{c} \beta \mathrm{s} \alpha}{2}-\frac{\sqrt{3}}{2} \mathrm{~s}^{2} \alpha \mathrm{s} \beta, \quad b=\frac{\sqrt{3}}{2} \mathrm{c} \beta \mathrm{s} \alpha-\frac{1}{2} \mathrm{~s}^{2} \alpha \mathrm{s} \beta, \\
c=\mathrm{c} \alpha \mathrm{s} \alpha \mathrm{s} \beta, \quad d=-\frac{1}{2} r_{b} \mathrm{c} \alpha \mathrm{s} \alpha \mathrm{s} \beta, \\
e=\frac{\sqrt{3}}{2} r_{b} \operatorname{c} \alpha \mathrm{s} \alpha \mathrm{s} \beta, \quad f=r_{b} \mathrm{c} \beta \mathrm{s} \alpha
\end{gathered}
$$

The motion screws are reciprocal to $S^{\mathrm{r}}$ and the basis of screw system can be calculated from Eq. (19), that is

$$
\boldsymbol{S}_{\mathbf{f}}=\left\{\begin{array}{l}
\mathbf{\$}_{f 1}=\left[\begin{array}{llllll}
1 & 0 & 0 & 0 & 0 & 0
\end{array}\right]^{\mathrm{T}} \\
\mathbf{\$}_{f 2}=\left[\begin{array}{llllll}
0 & 1 & 0 & 0 & 0 & 0
\end{array}\right]^{\mathrm{T}} \\
\mathbf{\$}_{f 3}=\left[\begin{array}{llllll}
0 & 0 & 1 ; & 0 & 0 & 0
\end{array}\right]^{\mathrm{T}}
\end{array}\right\}
$$

Hence, the 3(rA-4) $\mathrm{P}(\mathrm{rA}-3)$ parallel mechanism has pure rational DOF.

\subsubsection{Reconfiguration to Mobility 2}

By altering the phase of limb 3 to the phase of (rA-3) P(rA3 ) and other limb maintaining ( $\mathrm{rA}-6) \mathrm{P}(\mathrm{rA}-1)$, the metamorphic parallel mechanism evolved into a configuration with mobility 2, as shown in Figure 15.

Thus the constraint system of the mechanism is given as

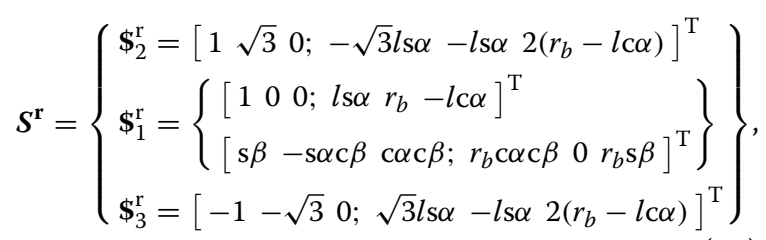

The motion screws are reciprocal to $S^{\mathrm{r}}$ and the screw system basis can be evaluated from Eq. (21), that is

$$
\boldsymbol{S}_{\mathbf{f}}=\left\{\begin{array}{l}
\boldsymbol{\$}_{f 1}=\left[\begin{array}{llllll}
1 & 0 & 0 & 0 & 0 & 0
\end{array}\right]^{\mathrm{T}} \\
\mathbf{\$}_{f 2}=\left[\begin{array}{llllll}
0 & 1 & 0 ; & 0 & 0 & 0
\end{array}\right]^{\mathrm{T}}
\end{array}\right\},
$$

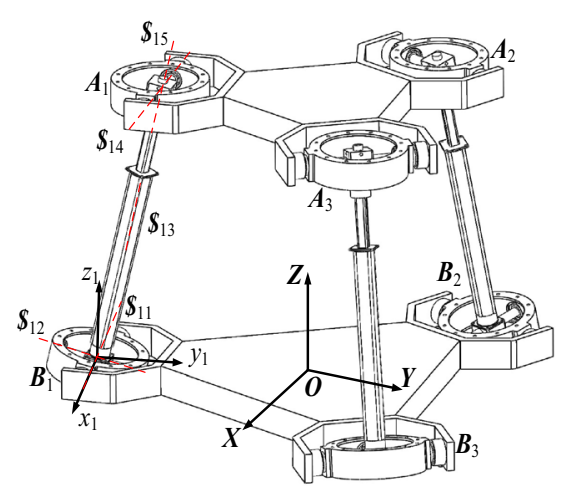

Figure 14. $3(\mathrm{r} A-4) \mathrm{P}(\mathrm{r} A-3)$ with pure rotation

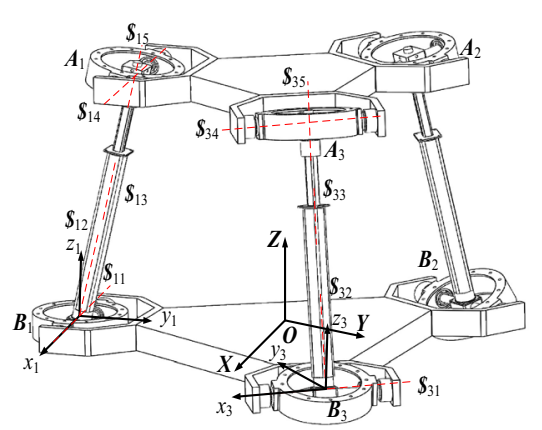

Figure 15. $1(r \mathrm{r}-3) P(r \mathrm{~A}-3)-2(r \mathrm{~A}-6) \mathrm{P}(\mathrm{r} A-1)$ 


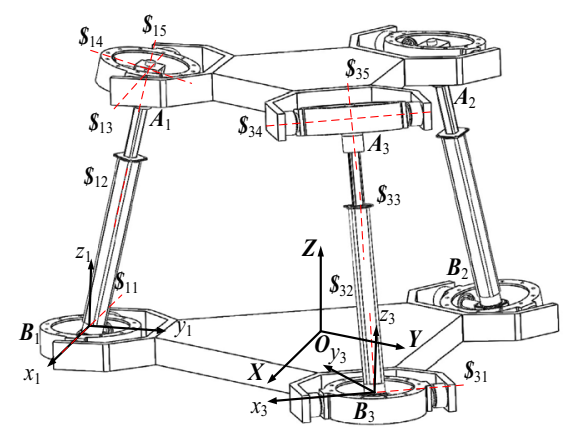

Figure 16. $2(r \mathrm{~A}-3) \mathrm{P}(\mathrm{rA}-3)-1(\mathrm{rA}-6) \mathrm{P}(\mathrm{rA}-1)$

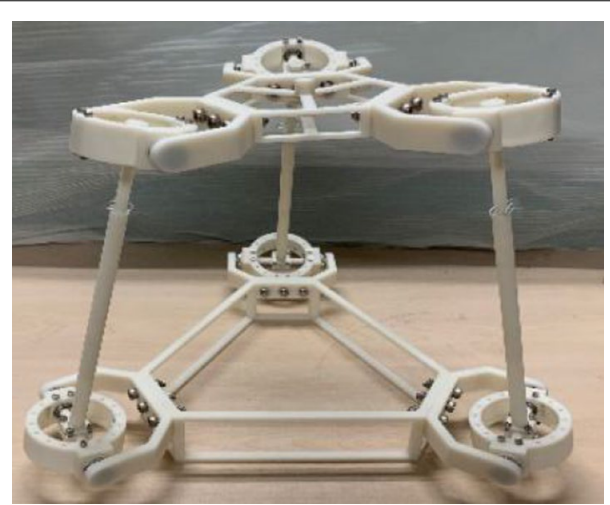

Figure 17. Physical prototype of $3(r A) P(r A)$

This indicates that the platform has 2 DOF with translation along the $X$ and $Y$ axes.

\subsubsection{Reconfiguration to Mobility 1}

The metamorphic parallel mechanism can be evolved into 1 DOF configuration by altering the upper and lower rA joints into rA-3 of the limb 1 and 2 and the limb 3 remain the phase of (rA-6)P(rA-1), as in Figure 16.

The constraint system of parallel mechanism with $2(\mathrm{rA}-3) \mathrm{P}(\mathrm{rA}-3)-1(\mathrm{rA}-6) \mathrm{P}(\mathrm{rA}-1)$ configuration is given as

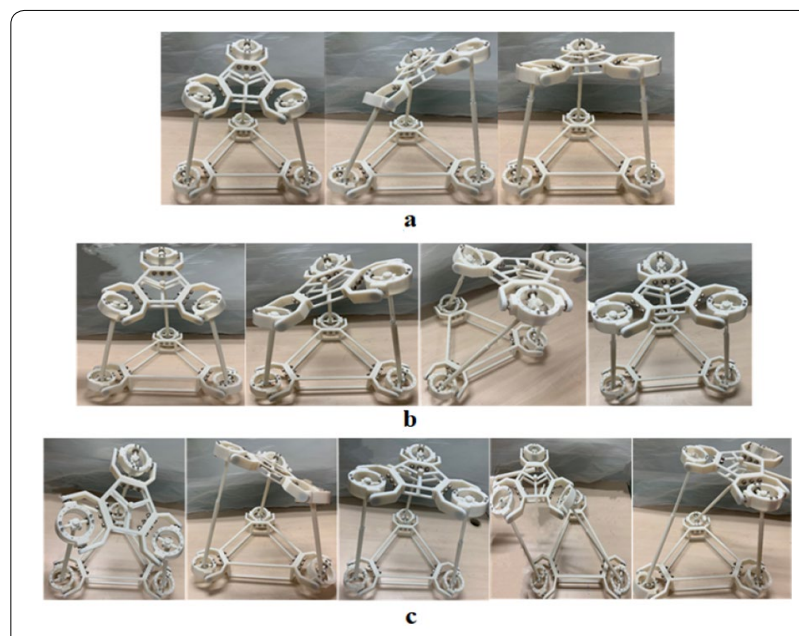

Figure 18. (a) $1 T 2 R$, (b) $3 T 1 R$, (c) $2 T 3 R$

Table 1. Phases and DOF of limb (rA)P(rA)

\begin{tabular}{llll}
\hline No. & $\begin{array}{l}\text { Equivalent } \\
\text { phase }\end{array}$ & Phase & DOF \\
\hline 1 & SPS & $1 \mathrm{P} 1,2 \mathrm{P} 1,2 \mathrm{P} 2$ & 6 \\
2 & UPS & $3 \mathrm{P} 1,3 \mathrm{P} 2,4 \mathrm{P} 1,4 \mathrm{P} 2,5 \mathrm{P} 1,5 \mathrm{P} 2$ & 6,5 \\
3 & RPS & $6 \mathrm{P} 1,6 \mathrm{P} 2$ & 54 \\
4 & UPU & $3 \mathrm{P} 3,3 \mathrm{P} 4,3 \mathrm{P} 5,4 \mathrm{P} 4,4 \mathrm{P} 5,5 \mathrm{P} 5$ & 5,4 \\
5 & UPR & $3 \mathrm{P} 6,4 \mathrm{P} 6,5 \mathrm{P} 6$ & 4 \\
6 & RPR & $6 \mathrm{P} 6$ & 3 \\
\hline
\end{tabular}

The motion screw system can be obtained by calculating the reciprocal screw of Eq. (23), and the basis of motion screw is given by

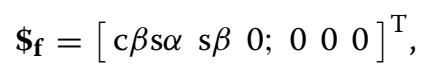

Thus the platform in the configuration of $2(\mathrm{rA}-3) \mathrm{P}(\mathrm{rA}-$ 3)-1(rA-6) $\mathrm{P}(\mathrm{rA}-1)$ has one revolute DOF.

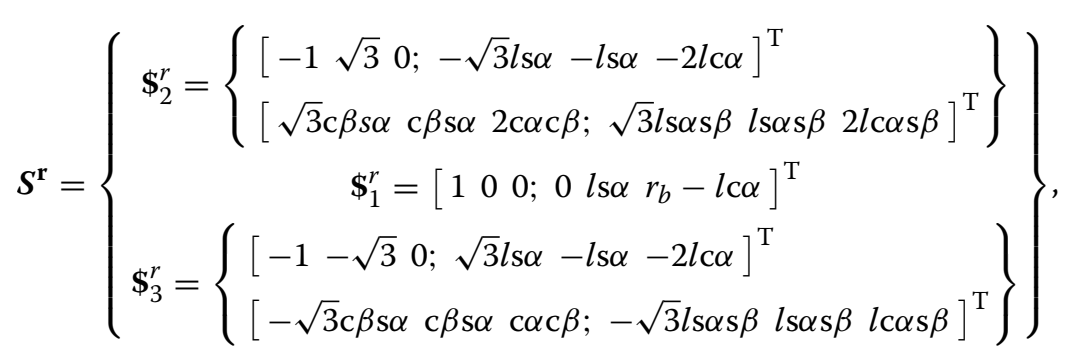


Table 2. The configurations of parallel mechanism $3(\mathrm{rA})$ $\mathbf{P}(\mathrm{rA})$

\begin{tabular}{|c|c|c|}
\hline No. & Equivalent configuration & Configuration \\
\hline 1 & $3 S P S$ & $3(1,2) P(1,2)$ \\
\hline 2 & 3UPS & $3(3,4,5) P(1,2)$ \\
\hline 3 & $3 R P S$ & $3(6) P(1,2)$ \\
\hline 4 & 3UPU & $3(3,4,5) P(3,4,5)$ \\
\hline 5 & $3 \mathrm{RPU}$ & $3(6) P(3,4,5)$ \\
\hline 6 & $3 R P R$ & $3(6) P(6)$ \\
\hline 7 & 2SPS-UPS & $2(1,2) P(1,2)-(3,4,5) P(1,2)$ \\
\hline 8 & 2SPS-RPS & $2(1,2) P(1,2)-(6) P(1,2)$ \\
\hline 9 & 2SPS-UPU & $2(1,2) P(1,2)-(3,4,5) P(3,4,5)$ \\
\hline 10 & 2SPS-UPR & $2(1,2) P(1,2)-(3,4,5) P(6)$ \\
\hline 11 & 2SPS-RPR & $2(1,2) P(1,2)-(6) P(6)$ \\
\hline 12 & 2UPS-SPS & $2(3,4,5) \mathrm{P}(1,2)-(1,2) \mathrm{P}(1,2)$ \\
\hline 13 & 2UPS-UPR & $2(3,4,5) P(1,2)-(3,4,5) P(1,2)$ \\
\hline 14 & 2UPS-RPS & $2(3,4,5) P(1,2)-(6) P(1,2)$ \\
\hline 15 & 2UPS-UPU & $2(3,4,5) P(1,2)-(3,4,5) P(3,4,5)$ \\
\hline 16 & 2UPS-RPR & $2(3,4,5) P(1,2)-(6) P(6)$ \\
\hline 17 & 2RPS-SPS & $2(6) P(1,2)-(1,2) P(1,2)$ \\
\hline 18 & 2RPS-UPS & $2(6) P(3,4,5)-(3,4,5) P(1,2)$ \\
\hline 19 & 2RPS-UPU & $2(6) P(1,2)-(3,4,5) P(3,4,5)$ \\
\hline 20 & 2RPS-UPR & $2(6) P(1,2)-(3,4,5) P(6)$ \\
\hline 21 & 2RPS-RPR & 2(6)P(1,2)-(6)P(6) \\
\hline 22 & 2UPU-SPS & $2(3,4,5) P(3,4,5)-(1,2) P(1,2)$ \\
\hline 23 & 2UPU-UPS & $2(3,4,5) P(3,4,5)-(3,4,5) P(1,2)$ \\
\hline 24 & 2UPU-RPS & $2(3,4,5) P(3,4,5)-(6) P(1,2)$ \\
\hline 25 & 2UPU-UPR & $2(3,4,5) P(3,4,5)-(3,4,5) P(6)$ \\
\hline 26 & 2UPU-RPR & $2(3,4,5) \mathrm{P}(3,4,5)-(6) \mathrm{P}(6)$ \\
\hline 27 & 2RPR-SPS & $2(6) P(6)-(1,2) P(1,2)$ \\
\hline 28 & 2RPR-UPS & $2(6) P(6)-(3,4,5) P(1,2)$ \\
\hline 29 & 2RPR-RPS & 2(6)P(6)-(6)P(1,2) \\
\hline 30 & 2RPR-UPU & $2(6) P(6)-(3,4,5) P(3,4,5)$ \\
\hline 31 & 2RPR-UPR & $2(6) P(6)-(3,4,5) P(6)$ \\
\hline 32 & SPS-UPS-RPS & $(1,2) P(1,2)-(3,4,5) P(1,2)-(6) P(1,2)$ \\
\hline 33 & SPS-UPS-UPU & $(1,2) P(1,2)-(3,4,5) P(1,2)-(3,4,5) P(3,4,5)$ \\
\hline 34 & SPS-UPS-UPR & $(1,2) P(1,2)-(3,4,5) P(1,2)-(3,4,5) P(6)$ \\
\hline 35 & SPS-UPS-RPR & $(1,2) P(1,2)-(3,4,5) P(1,2)-(6) P(6)$ \\
\hline 36 & SPS-RPS-UPU & $(1,2) P(1,2)-(6) P(1,2)-(3,4,5) P(3,4,5)$ \\
\hline 37 & SPS-RPS-UPR & $(1,2) P(1,2)-(6) P(1,2)-(3,4,5) P(6)$ \\
\hline 38 & SPS-RPS-RPR & $(1,2) P(1,2)-(6) P(1,2)-(6) P(6)$ \\
\hline 39 & SPS-UPU-UPR & $(1,2) P(1,2)-(3,4,5) P(3,4,5)-(3,4,5) P(6)$ \\
\hline 40 & SPS-UPU-RPR & $(1,2) P(1,2)-(3,4,5) P(3,4,5)-(6) P(6)$ \\
\hline 41 & SPS-UPR-RPR & $(1,2) P(1,2)-(3,4,5) P(6)-(6) P(6)$ \\
\hline 42 & UPS-RPS-UPU & $(3,4,5) P(1,2)-(6) P(1,2)-(3,4,5) P(3,4,5)$ \\
\hline 43 & UPS-RPS-UPR & $(3,4,5) P(1,2)-(6) P(1,2)-(3,4,5) P(6)$ \\
\hline 44 & UPS-RPS-RPR & $(3,4,5) P(1,2)-(6) P(1,2)-(6) P(6)$ \\
\hline 45 & UPS-UPU-UPR & $(3,4,5) P(1,2)-(3,4,5) P(3,4,5)-(3,4,5) P(6)$ \\
\hline 46 & UPS-UPU-RPR & $(3,4,5) P(1,2)-(3,4,5) P(3,4,5)-(6) P(6)$ \\
\hline 47 & UPS-UPR-RPR & $(3,4,5) P(1,2)-(3,4,5) P(6)-(6) P(6)$ \\
\hline 48 & RPS-UPU-UPR & (6) $P(1,2)-(3,4,5) P(3,4,5)-(3,4,5) P(6)$ \\
\hline 49 & RPS-UPU-RPR & (6) $\mathrm{P}(1,2)-(3,4,5) \mathrm{P}(3,4,5)-(6) \mathrm{P}(6)$ \\
\hline 50 & RPS-UPR-RPR & (6) $P(1,2)-(3,4,5) P(6)-(6) P(6)$ \\
\hline 51 & UPU-UPR-RPR & $(3,4,5) P(3,4,5)-(3,4,5) P(6)-(6) P(6)$ \\
\hline
\end{tabular}

\section{Physical Prototype and Configuration's Enumeration of 3(rA)P(rA)}

The experimental prototype was fabricated by $3 \mathrm{D}$ printing as shown in Figure 17. By adjusting the phases of $\mathrm{rA}$ joints in the limbs, 3(rA)P(rA) parallel mechanism with different configuration is generated.

In order to verify the effectiveness of the above analysis, specific configurations such as $1 \mathrm{~T} 2 \mathrm{R}, 3 \mathrm{~T} 1 \mathrm{R}$, and 2T3R are selected as examples. As shown in Figure 18, the mobility of each configuration is displayed by the prototype.

Since the upper and lower rA joints can be freely altered, a number of parallel mechanisms with different mobility can be generated. According to the analysis of all limb phases, it reveals a total of $C_{6}^{1} \cdot C_{6}^{1}=36$ types of equivalent limb phases as shown in the Table 1.

The equivalent configurations of assembled metamorphic parallel mechanism reveals a total of $C_{6}^{1}+C_{6}^{3}+5 \cdot C_{5}^{1}=51$ combinations as shown in the Table 2.

\section{Conclusions}

1. This paper presents a newly reconfigurable Axes joint inspired by the Rubik's Cube for the construction of metamorphic parallel mechanisms with the ability of mobility change. This rA joint is designed by two constraint methods of geometric and physical locking, and its six equivalent motion phases and corresponding mobility are analyzed.

2. Seven kinds of metamorphic parallel mechanisms are selected as representatives, and their constraints and mobility are analyzed. The degree of freedom of these seven metamorphic parallel mechanisms can vary from 6 to 1 . The biggest advantage of this $3(\mathrm{rA}) \mathrm{P}(\mathrm{rA})$ parallel mechanism is that the configuration of the rA joint can be adjusted to change the configuration of the parallel mechanism without disassembling the structure.

3. According to the analysis of the screw system of the variable limbs and parallel mechanisms, 36 kinds of limb phases and 51 kinds of parallel mechanism configurations are enumerated respectively.

4. The mobility of the selected configuration is verified by the prototype, which is consistent with the previous theoretical analysis.
Acknowledgements

Not applicable. 


\section{Authors' Contributions}

$D L$ was in charge of the whole trial; PJ wrote the manuscript; JL assisted with sampling and laboratory analyses. DZ and XK provided guidance and discussion in theory. All authors read and approved the final manuscript.

\section{Authors' Information}

Duanling Li born in 1974, is currently an professor at Beijing University of Posts and Telecommunications, China. She received her Ph.D. degree from Beihang University, China, in 2003. Her main research interests include mechanisms and robotics.

Pu Jia born in 1990, is currently a Ph.D. candidate at Automation School, Beijing University of Posts and Telecommunications, Beijing. His main research interests include mechanisms and robotics.

Jiazhou Li born in 1992, is currently a master candidate at Automation School, Beijing University of Posts and Telecommunications, Beijing, China. His main research interests include mechanisms and robotics.

Dan Zhang is currently a professor in the Department of Mechanical Engineering of the, Lassonde School of Engineering at York University. He received his Ph.D. degree in 2000 from Laval University, Canada. His research interests include robotics and mechatronics, high performance parallel roboticmachine development, micro/nano manipulation and MEMS devices, rehabilitation robot and rescue robot.

Xianwen Kong is an associate professor and programme director of M.Sc in Robotics at Heriot-Watt University, UK and a member of Edinburgh Centre for Robotics, a joint venture between Heriot-Watt University and the University of Edinburgh. His research interests include mechanisms and robotics focusing on the creative design of parallel manipulators with their applications in manufacturing and renewable energy.

\section{Funding}

Supported by National Natural Science Foundation of China (Grant No. 51775052), Natural Science Basic Research Plan in Shaanxi Province of China (Grant No. 2019JM-181), and Beijing Municipal Key Laboratory of Spaceground Interconnection and Convergence of China.

\section{Competing Interests}

The authors declare that they have no competing interests.

\section{Author Details}

${ }^{1}$ School of Automation, Beijing University of Posts and Telecommunications, Beijing 100876, China. ${ }^{2}$ College of Mechanical and Electrical Engineering, Shaanxi University of Science and Technology, Xi'an 712000, China. ${ }^{3}$ Beijing Post Intelligent Equipment Engineering Technology Research Center, Beijing 100876, China. ${ }^{4}$ Department of Mechanical Engineering, York University, Toronto, Canada. ${ }^{5}$ School of Engineering and Physical Sciences, Heriot-Watt University, Edinburgh EH14 4AS, UK.

Received: 16 December 2019 Revised: 15 October 2020 Accepted: 21 October 2020

Published online: 09 November 2020

\section{References}

[1] KWohlhart. Kinematotropic linkages. Recent Advances in Robot Kinematics. Kluwer, Dordrecht, The Netherlands, 1996: 359-368.

[2] J S Dai, R Jones. Mobility in metamorphic mechanisms of foldable/ erectable kinds. Proceedings 25th ASME Biennial Mechanisms and Robotics Conference, Atlanta, GA, Sept, 1998.

[3] LP Zhang, D L Wang, J S Dai. Biological Modeling and Evolution Based Synthesis of Metamorphic Mechanisms. Journal of Mechanism Design, 2008, 130(7): 32767.

[4] G GOGU. Branching singularities in kinematotropic parallel mechanisms. Proceedings of the 5th International Workshop on Computational Kinematics, Duisburg, Germany, 2009.
[5] XW Kong, J Yu. Type synthesis of 2-DOF 3-4R parallel mechanisms with both spherical translation mode and sphere-on-sphere rolling mode. ASME Journal of Mechanism Design, 2015, 7(4): 041018.

[6] XW Kong, Y Jin. Type synthesis of 3-DOF multi-mode translational/spherical parallel mechanisms with lockable joints. Mechanism and Machine Theory, 2016, 96: 323-333.

[7] XW Kong. Reconfiguration analysis of a 4-DOF 3-RER parallel manipulator with equilateral triangular base and moving platform. Mechanism and Machine Theory, 2016, 98: 180-189.

[8] D M Gan, J S Dai, Q Z Liao. Mobility change in two types of metamorphic parallel mechanisms. Journal of Mechanisms and Robotics, 2009, 1(4): 041007.

[9] D M Gan, J S Dai, J Dias, et al. Variable motion/force transmissibility of a metamorphic parallel mechanism with reconfigurable 3T and 3R motion. ASME 2015 International Design Engineering Technical Conferences and Computers and Information in Engineering Conference, 2015.

[10] D M Gan, J S Dai, L D Seneviratne. Unified kinematics and optimal design of a 3 rrps metamorphic parallel mechanism with a reconfigurable revolute joint. Mechanism and Machine Theory, 2016, 96: 239-254.

[11] D M Gan, J S Dai. Reconfiguration and static joint force variation of a 3rRps metamorphic parallel mechanism with 3R and 1T2R motion. Advances in Reconfigurable Mechanisms and Robots, 2015: 213-222.

[12] D M Gan, J S Dai, J Dias. Variable motion/force transmissibility of a metamorphic parallel mechanism with reconfigurable $3 T$ and $3 R$ motion. Journal of Mechanisms and Robotics, 2015, 8(5): 051001

[13] KT Zhang, J S Dai, Y F Fang. Topology and constraint analysis of phase change in the metamorphic chain and its evolved mechanism. Journal of Mechanical Design, 2010, 132(12): 121001.

[14] KT Zhang, J S Dai. Reconfiguration of the plane-symmetric double-spherical $6 \mathrm{R}$ linkage with bifurcation and trifurcation. Journal of Mechanical Engineering Science, 2016: 203-210.

[15] KT Zhang, J S Dai. Screw-system-variation enabled reconfiguration of the bennett plano-spherical hybrid linkage and its evolved parallel mechanism. Journal of Mechanical Design, 2015, 137(6): 062303.

[16] M C Palpacelli, L Carbonari, G Palmieri. Details on the design of a lockable spherical joint for robotic applications. Journal of Intelligent \& Robotic Systems, 2016, 81(2): 169-179.

[17] W Ye, Y F Fang, KT Zhang. Mobility variation of a family of metamorphic parallel mechanisms with reconfigurable hybrid limbs. Robotics and Computer-Integrated Manufacturing, 2016, 41: 145-162.

[18] W Ye, Y F Fang, S Guo. Design and analysis of a reconfigurable parallel mechanism for multidirectional additive manufacturing. Mechanism and Machine Theory, 2017, 112: 307-326.

[19] W Ye, Chai X, KT Zhang. Kinematic modeling and optimization of a new reconfigurable parallel mechanism. Mechanism and Machine Theory, 2020: 149.

[20] B Wang, Y F Fang. Type synthesis of metamorphic parallel mechanism with spherical five bar linkage. Journal of Mechanical Engineering, 2018, 54(19): 19-26. (in Chinese)

[21] H B Qu, S Guo. Topology and mobility variations of a novel redundant reconfigurable parallel mechanism. Mechanisms and Machine Science, 2016, 36: 223-233.

[22] H B Wang, ZY Qi, ZW Hu, et al. Application of parallel leg mechanisms in quadruped/biped reconfigurable walking robot. Journal of Mechanical Engineering, 2009, 45: 24-30. (in Chinese)

[23] CXTian, Y F Fang, S Guo. A class of reconfigurable parallel mechanisms with five-bar metamorphic linkage. Journal of Mechanical Engineering Science, 2017, 231: 2089-2099.

[24] CX Tian, Y F Fang, S Guo. Structure synthesis of reconfigurable parallel mechanism with closed-loop metamorphic linkages. ARCHIVE Proceedings of the Institution of Mechanical Engineers Part C Journal of Mechanical Engineering Science, 2017, 203-210: 1-14.

[25] Y Q Song, X S M, J S Dai. A novel 6R metamorphic mechanism with eight motion branches and multiple furcation points. Mechanism and Machine Theory, 2019: 142. 